\title{
A Review Article on Internet-based Psychological Interventions in Primary Care. What Is the Global Experience? How Reliable Are Results from RCTs? Lessons Learned from the European, US and Australian Case Studies $^{1}$
}

\author{
Melita Sogomonjan \\ Tanel Kerikmäe \\ Tallinn Law School, \\ Tallinn University of Technology \\ Akadeemia tee 3 , \\ Tallinn 12618, Estonia \\ E-mail: melita.sogomonjan@taltech.ee \\ E-mail: tanel.kerikmae@taltech.ee \\ Pille Ööpik \\ Department of Family Medicine, \\ Faculty of Medicine, \\ University of Tartu \\ L. Puusepa 1a-2120, \\ Tartu, Estonia \\ E-mail: pille.oopik@ut.ee
}

Abstract: In the context of the EU's Digital Single Market (eHealth) Strategy, the deployment of digital tools for patients'empowerment and personcentred care is of high demand and importance. Shifting from treatment to health promotion and disease prevention, a variety of internet-based cognitive behavioural therapy programmes have been proven to be effective for managing common mental health disorders in secondary care even hough the effectiveness and the clinical use of internetbased cognitive behavioural therapy programmes alone in primary care have not been approved yet. Additionally, such interventions are neither included in the international clinical guidelines for treating common mental health disorders nor regulated by Member States as a

This work was supported by the Estonian Research Council grant PUT 1628. 
healthcare service. Despite that, the UK National Health Service and the Swedish National Board of Health and Welfare endorse the use of internet-based cognitive behavioural therapy as a first treatment option. The aim of this research is to investigate the global experience of internet-based cognitive behavioural therapy programmes in controlled and real-life conditions in general practice and to evaluate the reliability of the results and concomitantly their compliance with the European Commission's eHealth Strategy. A systematic review of quantitative studies was conducted from January 2007 to December 2017. The results indicated that unsupported internet-based cognitive behavioural therapy programmes alone are less effective than combined therapy options for treatment purposes, if no additional therapy is prescribed. Guided internet-based cognitive behavioural therapy may supplement traditional treatment methods resulting in improving the control of mental disorders, but are unable to demonstrate consistent quality or replace face-to-face therapy.

Keywords: anxiety, depression, digital single market, effectiveness, general practice, internet-based cognitive behavioural therapy, randomized control trial

\section{Introduction}

Common mental health disorders have significant adverse impacts on the societal and emotional functioning of individuals (Collins et al., 2018). At a global level, in 2015 the prevalence of depression was estimated to be $4.4 \%$, while $3.6 \%$ of people suffered from anxiety disorders (WHO, 2017). Access to healthcare for people experiencing these common mental disorders is a challenge mainly due to lack of resources reflected in the limited number of qualified therapists and the health system financing (NICE, 2011; WHO, 2017; Layard \& Clark, 2014). Given that, the digital single market sets a priority to empower citizens with digital health solutions in order to foster the integration of health promotion and prevention into primary care. Digital transformation of healthcare services is aimed to develop new care models that will pursue with the delivery of efficient and cost-effective care. (Communication COM(2018) 233 final)

According to Berger et al. (2016) one way to increase access to evidence-based psychological treatment in primary care is to provide internet- or computer-based cognitive-behavioural treatment (iCBT/cCBT). $\mathrm{iCBT}$ or $\mathrm{cCBT}$ is a low-intensity 
non-pharmacological intervention that includes internet-delivered psychological therapies or any downloadable software, online multimedia programme or smartphone application designed to teach users the basic concepts of cognitive behavioural therapy and the skills for managing their mental health symptoms (Jonassaint et al., 2017; Pennant et al., 2015; Marks et al., 2007). Despite the variety of iCBT interventions available on the global market, the most widely known internet-based psychotherapies used so far include the Beating the Blues, MoodGYM and Colour Your Life programmes. iCBT has the potential to increase access to high-quality mental health treatment for minority populations Jonassaint et al., 2017). Large-scale randomized control trials (RCTs) have investigated the high level of adherence and significant outcomes in reducing symptoms of depression considering guided therapist-assisted computerized cognitive behavioural therapies in routine clinical practice (Jonassaint et al., 2017; Richards \& Richardson, 2012; Perini et al., 2009; Ruwaard et al., 2009; Andersson et al., 2005; Berger et al., 2011; Johansson et al., 2012; Knowles et al., 2014; Andersson \& Cuijpers, 2009; Kay-Lambkin et al., 2011).

The efficacy of iCBT for depression in primary care so far has been investigated by only one study, wherein iCBT has been shown to be more effective than treatment-as-usual (Proudfoot et al., 2004). Salomonsson et al. (2017) have argued that using a stepped-care model ${ }^{2}$ is an effective and efficient way to achieve improvements for nearly two thirds of primary care patients with common mental disorders while using fewer therapist resources.

Concerns about the clinical effectiveness of mental health services provided through internet-based interventions incited controversy among health care professionals worldwide (Montero-Marín et al., 2015; Du et al., 2013). The ambiguity regarding the effectiveness of iCBT programmes in treating depression and anxiety is apparent. If iCBT programmes are effective for use in real-life conditions in general practice, such programmes should not meet barriers to uptake or demonstrate high attrition rate.

Therefore, the aim of this review is to evaluate the effects and adherence of iCBT for adults with depression and anxiety disorders in general practice. The following main questions are addressed to complete this review:

1. What is the global experience of iCBT in primary care?

2. Does iCBT work in the 'real world' in general practice?

3. How reliable are the results from RCTs?

2 Stepped care model: (Step I = guided self-help CBT + Step II = face-to-face CBT or continued guided self-help treatment) 


\section{Methods}

\subsection{Search strategy and study selection}

A comprehensive search was carried out on Scopus, the Web of Science, PubMed and Psychology Medicine electronic databases from January 2007 to December 2017 in order to retrieve the most relevant data for current versions of iCBT. The search terms were 'internet- or computer-based cognitive-behavioural therapy', 'primary care', 'randomized control trials', 'efficacy', 'depression' and 'anxiety'. Articles were selected by title, by abstract or by reading the full original paper. Only articles in English were included.

\subsection{Eligibility criteria}

For the purpose of this study we selected RCTs evaluating the efficacy and, where possible, the effectiveness of iCBT for depression and anxiety disorders in primary care. Studies were eligible for inclusion if RCTs were performed in the primary care setting to investigate the comparison between:

(1) guided iCBT with minimum therapist contact with the control group or a wait-list control (WLC) group and

(2) unguided self-help programmes with face-to-face therapy.

The exclusion criteria were non-randomized control trials or studies that recruited children and adolescents or patients with severe mental health disorders including psychiatric co-morbidity, psychosis, bipolar disorder, personality disorders, alcohol or drug dependence or suicidal tendencies. Studies with an economic evaluation, including those evaluating the cost-effectiveness and efficiency of iCBT, were excluded from the analysis; however, they are considered important for further research.

\subsection{Quality assessment}

The methodological quality of studies has been evaluated, including criteria for intervention trials. No formal assessment of quality was performed as the main search strategy focused on whether the trial was randomized and performed in a primary care setting. 


\section{Results}

\subsection{Description of studies}

A systematic review of 510 research articles were retrieved from the PubMed ( $n$ $=37)$, Scopus $(n=37)$, Web of Science $(n=53)$ and Psychological Medicine $(n=$ 383) databases. As shown in Figure 1, the literature search identified 64 duplicate studies, and 410 further studies were removed for the reason that they did not address the scope of the current research. For more detailed evaluation 36 studies were considered. Only 14 studies met the inclusion criteria. Of those 14 studies,

Figure 1. Identification of studies for review

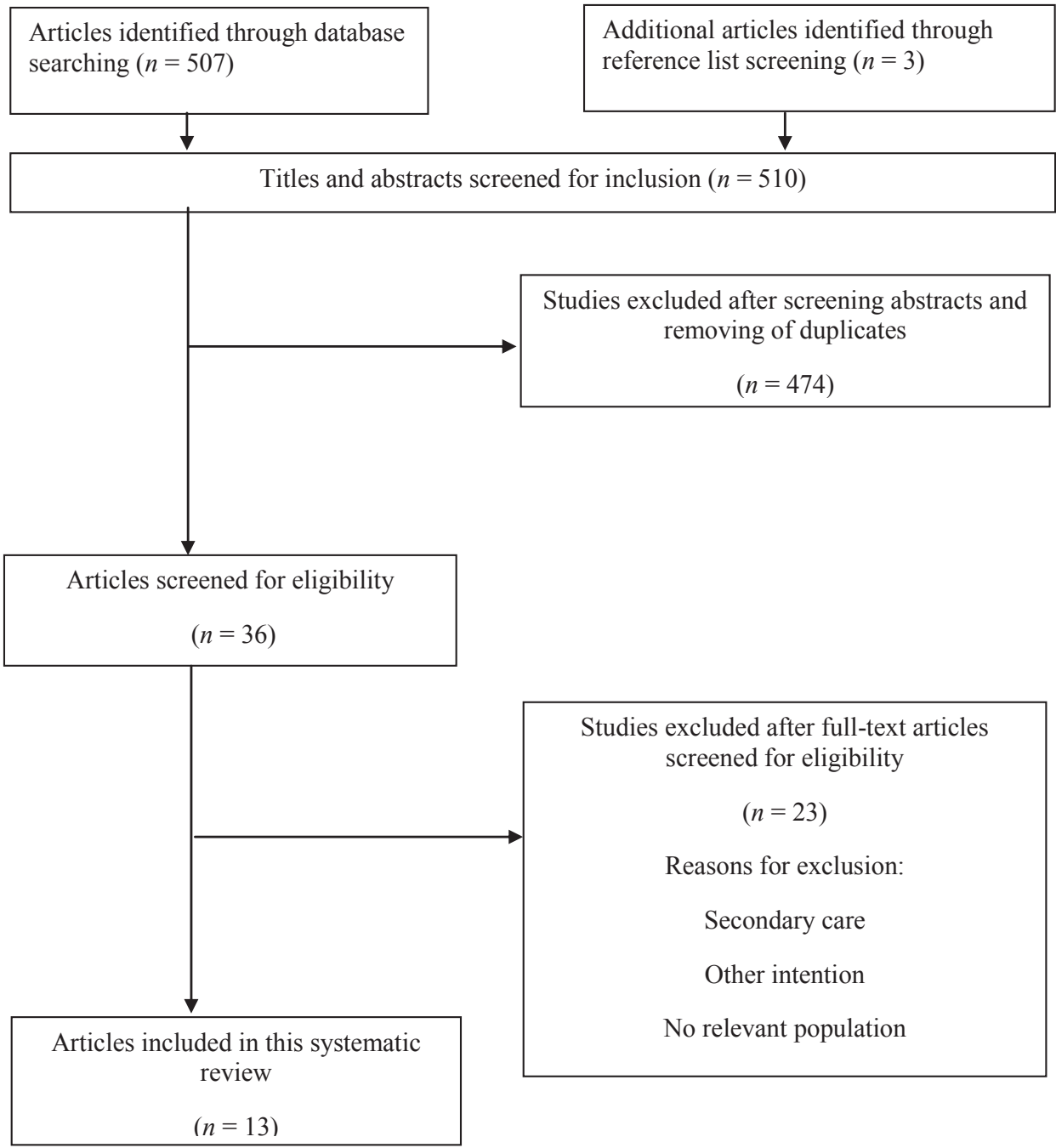


one study was removed due to insufficient data. All 13 studies were quantitative and conducted in Europe (69.2\%), USA (15.4\%) and Australia (15.4\%).

\subsection{Participants}

Participants included in the studies were prescribed iCBT by their general physicians (GP) or licensed psychologists. The participants' mean age in the studies varied from 33.7 to 45.2, except in the study by Hickie et al. (2010) where patients were recruited starting from age 16. In all studies female participants constituted the overwhelming majority.

Of the 4,265 adult primary care patients, depression was estimated among 1,589 patients. The majority of patients participating in RCTs experienced several types of anxiety disorders $(\mathrm{n}=2,676)$, including generalized anxiety disorder $(\mathrm{n}=1,129)$, social anxiety disorder $(\mathrm{n}=75)$, post-traumatic stress-disorder $(\mathrm{n}=$ $181)$, panic disorder with or without agoraphobia $(n=715)$ and other anxietyrelated conditions $(n=575)$. Out of 8,401 participants assessed for eligibility, 3,134 patients remained until post-treatment. All in all, 2,717 patients received internet-based psychological therapy (both guided and unguided iCBT). Additionally, 264 patients were enrolled in the iCBT course after the treatment group had completed the programme. Treatment and control groups were followed up with at three, four, six, eight and twelve months post-treatment, depending on the study design. A variety of measurement tools were used to assess patients at baseline and post-treatment and during the follow-up period.

\subsection{The global experience of iCBT in primary care}

A literature review of randomized controlled trials of iCBT in primary care in Europe, the USA and Australia proved that additional professional support is required to engage depressed patients with self-help internet-based psychotherapies. At present, computerized CBT is offered by many healthcare systems as a minimally supported low-intensity psychological intervention and as part of a stepped-care framework (Salomonsson et al., 2017; Høifødt et al., 2013). The level of support for each patient is different and it varies mainly in severity of depression and the patient's ability to perform tasks online. Even though enhancement in the level of support and guidance increase uptake and effectiveness (Andersson \& Cuijpers, 2009; Baumeister et al., 2014), evidence has accumulated to indicate that a low-intensity form of support such as technical telephone support had no additional clinical benefits when iCBT was added to usual primary care (Gilbody et al., 2017). 


\subsection{Drop-out and participants' adherence to iCBT throughout selected studies}

In the included studies, iCBT modules aimed to help patients gain mastery over their symptoms using CBT techniques. Out of 3,134 patients remaining at posttreatment, 484 patients completed all sessions. The mean number of completed sessions varies from 1.1 to 7.2 with an estimated number of 4.1. Participants in the intervention group had a relatively higher drop-out rate relative to participants in the wait-list control group (Høifødt et al., 2013). Even though the drop-out rate was related to improvement attributed to other concurrent treatments (Newby et al., 2013), a variety of adverse events was also reported during treatment. As a consequence of commencing iCBT, the patients experienced a negative impact on their general wellbeing and reported slightly worse mood and more anxiety (Salomonsson et al., 2017; Rollman et al., 2017; Simblett et al., 2017).

In the studies conducted by de Graaf et al., in 2009 and in 2011, participants completed the mean of 3.4 sessions of Colour Your Life in the iCBT group and 4.0 in the iCBT plus treatment-as-usual group (de Graaf et al., 2009; de Graaf et al., 2011).

Altogether, 1,507 participants were allocated to participate in the Beating the Blues programme. In the study by Kessler et al. (2009) in the iCBT group, at the four-month follow-up 90 participants completed five sessions, while at the eight-month follow-up 70 participants completed eight sessions (Kessler et al., 2009). In the study by Gilbody et al. (2015), 18\% (31 of 175) of participants completed all eight sessions. Moreover, 44\% (93 of 210) of participants reported non-serious adverse events and 7\% (15 of 210) reported serious adverse events (Gilbody et al., 2015). In the study by Rollman et al. (2017), at the six-month follow-up, $36.7 \%$ (221 of 603) of participants completed all eight sessions of the programme. The mean of 6.3 sessions of the BtB programme (40\%) in the iCBT group and the mean of 7.2 sessions (32\%) in the computerized cognitive remediation therapy (cCRT) group were completed in the study by Simblett et al. (2017). In the study by Hickie et al. (2010), 41\% (12 of 29) participants did not complete MoodGYM programme. In the study by Gilbody et al. (2015), trained technicians made weekly telephone calls to 242 patients using MoodGYM. Only 16\% (29 of 186) of participants completed all six sessions of the MoodGYM programme. In addition, $45 \%$ of participants reported adverse events (93 participants demonstrated non-serious adverse events and 15 participants reported serious adverse events). In the minimally supported group, $10.4 \%$ of participants completed all five sessions of MoodGYM and $19.4 \%$ of participants completed the telephone-facilitated iCBT. (Gilbody et al., 2017) In 
the study by Høifødt et al., 2013 out of 52 participants in the intervention group, 31 participants $(60 \%)$ adhered to the MoodGYM programme and attended 7 sessions. A mean of 3.8 of the 5 modules was completed during the treatment period by 50 patients. A mean of 7.2 sessions were attended. At seven weeks post-treatment, $15 \%$ participants $(\mathrm{n}=8)$ had completed the treatment. However, $45 \%$ of participants $(n=19)$ in the intervention group received additional treatment during the six-month follow-up period. (Høifødt et al., 2013)

In the first study by Newby et al. (2013), 89\% (41 of 46) of participants in the iCBT group completed all sessions of the Worry and Sadness Program. In the second study $41.2 \%$ (56 of 136) of participants completed all six lessons. (Newby et al., 2013)

In the study by Kivi et al. (2014), the supported iCBT Depressionshjälpen programme was provided for 44 depressed patients. In twelve weeks, 20 of $36(56 \%)$ participants completed all seven modules and the average number of completed sessions was 5.1 . Only $10 \%$ of participants reported adverse events. (Kivi et al., 2014)

In addition to treatment-as-usual, a supported Wellness Workshop CD-ROM programme was provided for 100 participants in the study by Levin et al. (2011). After two weeks, participants received a brief telephone call from the psychologist, but 27 participants did not start the program. The programme was used by 72 participants for an average of 2.9 weeks. (Levin et al., 2011)

In the study by Berger et al. (2017), the mean number of completed modules of the Velibra programme was 3.9. Thirty-two participants (45.7\%) completed all six modules of the programme. Eight participants (11.4\%) did not start the programme at all. (Berger et al., 2017)

\subsection{Effects of the internet-based interventions}

The results showed no clinically or statistically significant changes before treatment and immediately after treatment between treatment groups in all studies (Berger et al., 2017; Høifødt et al., 2013; Newby et al., 2013; Rollman et al., 2017; Simblett et al., 2017; Gilbody et al., 2017; de Graaf et al., 2009; Levin et al., 2011; de Graaf et al., 2011; Hickie et al., 2010; Kessler et al., 2009; Gilbody et al., 2015). Less than half of the patients recovered at post-treatment. Results from the studies by Høifødt et al. (2013) and Rollman et al. (2017) demonstrated that mean scores for the face-to-face group and WLC group were lower compared with scores for the intervention group for the depressive and 
anxiety subscales at post-treatment and at the six-month follow-up. Kivi et al. (2014) found no significant differences between the reduction in scores for the iCBT and TAU groups during treatment or post-treatment. Berger et al. (2017) emphasized in their results that, at post-treatment, the participants benefited significantly in the TAU group plus unguided iCBT more, compared with the TAU group only. In the study by Simblett et al. (2014), participants in all groups demonstrated a decrease in symptoms of distress across time, but there was very little difference in terms of functional ability at post-treatment. Clinically significant improvement was noted in the study by de Graaf et al. (2009) for 26 patients in the iCBT group from 91 patients available at post-treatment. At the twelve-month follow-up, 36 patients in the intervention group were determined to be in remission (de Graaf et al., 2009). Statistically significant differences on recovery were found in the studies by Hickie et al. (2010) and Levin et al. (2011). Participants in the intervention group demonstrated significant decreases in dysfunctional attitudes and were more likely to go into remission than participants in the TAU group (Levin et al., 2011). A small, clinically meaningful difference was found immediately after treatment in favour of enhanced GP care plus the MoodGYM group (Hickie et al., 2010). A reliable improvement in reducing depression and anxiety disorders were demonstrated in the studies by Hickie et al. (2010) and Newby et al. (2013). A small difference was found in favour of the MoodGYM programme vs usual GP care at the twelve-month follow-up (Gilbody et al., 2015). A significant between-group difference was found in the study by Gilbody et al. (2017) in anxiety scores in favour of telephone-facilitated iCBT.

\subsection{The impact of iCBT work in a 'real-world' general practice}

A 'real-world' general practice significantly differs from develop-led trials, where additional professional support was included and the effects of the internet-based psychotherapies were measured under strictly controlled conditions. Studies delivering $\mathrm{iCBT}$ in real-life conditions in primary care are limited and have been absent for a long time. The impact of iCBT work on depression outcome in reallife conditions was evaluated in our review only in the 2014 study by Kivi et al. Licensed psychologists and psychotherapists $(\mathrm{n}=12)$ were involved to support patients randomized for iCBT. Even though the proportion of recovered patients between treatment groups was similar $(n=15, \mathrm{BDI}-\mathrm{II} \leq 13)$, eight patients in the iCBT group at post-treatment were undergoing pharmacological therapy and three patients deteriorated. (Kivi et al., 2014) 


\subsection{Therapist time}

This review includes five trials in which therapist support time has been measured. Therapist time varies from two minutes to 30 minutes depending on the study design. Kessler et al. (2009) found that the quality of care was higher when CBT was facilitated by a therapist in real-time monitoring. Furthermore, real-time online $\mathrm{CBT}$ was suggested for patients with severe symptoms (Kivi et al., 2014).

In the study by Gilbody et al. (2015), patients allocated to the BtB programme received on average 6.2 minutes of support calls from a technician and those in the MoodGYM group received 6.5 minutes. In the study by Newby et al. (2013), a clinician and therapist regularly spent on average 23.37 minutes per patient on email and telephone contact during the trial. After each module, in the study by Høifødt et al. (2013), participants received face-to-face support (a range of 15-30 minutes). In the study by Kivi et al. (2014), therapists spent an average of 15 minutes on emails or telephone calls with each patient during each week. Every two weeks participants received telephone calls lasting from 15 to 30 minutes or an email from care managers in the study by Rollman et al. (2017).

\subsection{Reliability of RCT results}

Even though a small dosage of iCBT was associated with lower antidepressant medication use (de Graaf et al., 2011), it is difficult to determine the effectiveness of iCBT insofar as patients were financially compensated and allowed to undergo other treatment.

\subsubsection{Financial compensation}

To minimize the attrition rate and the number of drop-outs, participants in the study by de Graaf et al. (2009) were compensated $€ 25$ for internet use. In the study by Levin et al. (2011), each participant received $\$ 75$ at each data point for completing the questionnaire and interview assessments. Finally, in the study by Rollman et al. (2013), after each completed assessment at three, six and twelve months, patients received from $\$ 15$ to $\$ 60$.

\subsubsection{Psychotropic medication}

No additional data have been collected about the impact of medication on patient outcomes while other interventions have been employed. In this review participants in all studies were equally likely to receive pharmacotherapy. In the 2010 study by Hickie et al., participants did not receive pharmacological 
care at the time of study participation. Results from studies demonstrated that participants in the iCBT group were more likely to receive antidepressants compared with patients in the usual care group or in the treatment plus usual care group. It is difficult to evaluate the size of effect and relation between medication, face-to-face treatment or iCBT as long as data about the use of pharmacotherapy pre- and post-treatment and during the follow-up period are not available, which is true for the studies by Gilbody et al. (2017), Simblett et al. (2017), Levin et al. (2011), Newby et al. (2013) and Berger et al. (2017). Moreover, no study has yet analysed the outperformance of unsupported iCBT or guided iCBT as compared to pharmacological treatment.

\section{Discussion}

\subsection{Main findings}

This review includes both primary and secondary outcomes on depressed patients in the intervention trials for internet-based psychotherapies. Within the last ten years, more than 500 studies have been published examining telepsychiatry. The results from recent trials demonstrated reduction of symptoms of depression and anxiety even though participants were more likely to continue the use of antipsychotic medication and usual care. It is obvious that additional therapy and regular guidance result in improved outcomes and control of mental disorders. It has been noted that treatment outcomes from supported iCBT immediately at post-treatment are more efficacious than in wait-list control conditions. However, the effectiveness of $\mathrm{iCBT}$ in such trials is diminished at follow-up. Although iCBT is aimed at treatment of mild to moderate symptoms of depression and anxiety, six studies in this review included participants with more severe symptoms. Participants recruited within the primary care setting by health care providers with experience in CBT were more likely to complete iCBT modules compared with self-referred participants and those recruited through media. As long as therapist input has been considered important in terms of participant engagement, our findings underline that a minimum of 30 minutes per week is required either face-to-face or through telephone calls. In the methodologically stronger studies, participants demonstrated a high attrition rate and low adherence to iCBT intervention. In those studies, the secondary outcome at follow-up was considered at least a six-month rather than at a threemonth follow-up or immediately at post-treatment. Our findings emphasize that the effectiveness of iCBT interventions in the primary care setting as an effective option for non-pharmacological therapy cannot be confirmed for 
several reasons. First, the secondary outcome of the trials has not been measured with a clinical interview to establish the presence of depression according to accepted classification systems. The 'effectiveness' and 'efficacy' of iCBT were evaluated on participants' self-reported measures (questionnaires/assessments). Second, participants were free to use medication prior to the beginning of the study, during the trial, post-treatment and at follow-up. Thirdly, unemployed participants in three studies received financial compensation. Eventually, high drop-out and attrition rates are presented in all studies where financial compensation was not offered. Even though participants underwent an informed consent that was approved by the Review Board, information about the approval from the medical ethics committee is missing, especially in the studies where patients were provided with financial compensation. In two studies, participants reported reasons for dropping out. Only a small number of participants in our review completed all sessions of iCBT (Berger et al., 2017; Høifødt et al., 2013; Newby et al., 2013; Rollman et al., 2017; Simblett et al., 2017; Gilbody et al., 2017; Kivi et al., 2014; de Graaf et al., 2009; Levin et al., 2011; de Graaf et al., 2011; Hickie et al., 2010; Kessler et al., 2009; Gilbody et al., 2015; Waller \& Gilbody, 2009). This, however, could not prove that the use of iCBT was effective in the reduction of depression and anxiety disorders insofar as data concerning reasons for drop-out are missing in most of the studies. In the end, information according to treatment fidelity (i.e. whether therapists who provided iCBT were supervised and treatment was provided by designed protocol) and security issues (the manner of treatment delivery) are absent in many studies.

\subsection{Strengths and limitations}

The current research was restricted to articles published in English within the last ten years. Researchers confirm that a risk of bias assessment could not be undertaken. Studies eligible for inclusion were primarily efficacy studies with lack of comparison groups evaluating the evidence for effectiveness of iCBT alone or for the added benefit of iCBT. In most studies, patients were recruited through primary care by licensed psychologists or neuropsychologists rather than by general physicians. In addition, programmes commercially available for a wider population are not tailored specifically for minority patients. Data about the cultural diversity of the populations studied have not been considered in most studies. 


\subsection{The interpretation of the study results in relation to existing literature}

A previous review found $\mathrm{iCBT}$ to be no less effective than face-to-face cognitive-behavioural therapy (Kaltenthaler et al., 2002; Kaltenthaler et al., 2004; Kaltenthaler et al., 2006) and to reduce therapist time (Kaltenthaler et al., 2002; Kaltenthaler et al., 2006). At present, the results demonstrated reduction of symptoms of depression and anxiety even though participants were more likely to continue the use of antipsychotic medication and usual care. Treatment outcomes from supported iCBT immediately at post-treatment are more efficacious than in WLC conditions. The effectiveness of $\mathrm{iCBT}$ in trials is diminished at follow-up.

\subsection{Compliance with digital single market strategy}

In the context of digital single market, integrated eMental Health applications into primary care increase the collaborative economy of Member States, in particular improving the complementarity of cross-border healthcare services. Data collected from internet-based psychological interventions help us achieve the second priority of the European Strategy — personalized medicine through shared European data infrastructure. Although it seems that the European Strategy interests are more directed at protecting economic interests rather than human rights to receive qualified health and care (see also Uusitalo, 2018). In fact, EU legislation on medical devices, data protection, electronic identification and security of network give a legal framework for the eHealth infrastructure. However, digital solutions for health prevention and control of disease are not yet legally defined as healthcare services either in the regulation of Member States or in the EU legislation. All this leads us to think how to evaluate state capacity to handle healthcare services in the context of human dignity (see also Kerikmäe \& Joamets, 2018; Kerikmäe et al., 2016).

\section{Conclusion}

Researchers are inclined to affirm that iCBT is used to control common mental health disorders enabling easy access to rather than providing quality of treatment. Reduced symptoms are likely to be an outcome due to reasons not considered in the studies. Engagement of patients with common mental health disorders with iCBT might show an effect but only if offered alongside a high level of facilitation and support or delivered online (Gilbody et al., 
2017; Kessler et al., 2009). The studies included in this review underlined the importance of additional resources in general practice. The specific impact of iCBT is difficult to determine without having care coach support and if data about the time therapists spent on each patient are not available. Instead, it makes it more complicated to evaluate which treatment option or a combination of therapeutic options resulted in the improved outcomes. Given the difficulty in assessing the clinical efficacy and effectiveness of iCBT, no study has revealed a drop-out rate between unemployed and employed participants or between participants on pharmacological therapy and those who did not take medication therapy. Researchers are inclined to confirm that patients completed iCBT modules either due to financial compensation or regular telephone calls and emails. Our findings underline that neither efficacy nor effectiveness of iCBT has been proven and should not replace face-to-face therapy until evidence for iCBT's benefits is found among service providers and patients. All this means that the European Strategy on enabling transformation of health and care in the digital single market does not meet directly patients' needs but is fostering sharing economic interests.

\section{Declaration of interest}

The authors report no conflicts of interest. The authors alone are responsible for the content and writing of the paper.

Melita Sogomonjan is a PhD student at Tallinn Law School, Tallinn University of Technology. Her research is focused on the development and regulation of eHealth Policy and Public Health Administration in the EU.

Tanel Kerikmäe, LLM, PhD, is a full professor of law and has been contributing to the research of European law and policies. He created a research group of law \& tech that received significant funding from the EU Commission (H2020, Jean Monnet Centre of Excellence), NATO, Folke Bernadotte Academy, USAID, etc. Many of these grants are focused on developing legal digital skills. Tanel has contributed to Springer, Ashgate, Kluwer, Oxford and Cambridge publishers and is a foreign member of the National Academy of Legal Sciences of Ukraine. The articles he has published include topics on EU legal policies, constitutional law, digital single market, automatization, legal impediments to new technologies, augmented and artificial intelligence in legal practice. 
Pille Ööpik, MD, PhD is a family physician and a researcher at the University of Tartu. She has more than 30 years of experience in clinical medicine. Her main research field is treatment of depression in family medicine. Since 1995, she is head of the Ädala Family Medicine Centre in Tallinn.

\section{References}

Andersson, G.; Bergström, J.; Holländare, F.; et al. (2005), 'internet-based self-help for depression: randomised controlled trial,' The British Journal of Psychiatry, vol. 187, no. 5, pp. 456-461. https://doi.org/10.1192/bjp.187.5.456

Andersson, G. \& Cuijpers, P. (2009), 'internet-based and other computerized psychological treatments for adult depression: a meta-analysis,' Cognitive Behaviour Therapy, vol. 38, no. 4, pp. 196-205. https://doi.org/10.1080/16506070903318960

Baumeister, H.; Reichler, L.; Munzinger, M. \& Lin, J. (2014), 'The impact of guidance on internet-based mental health interventions-a systematic review,' internet Interventions, vol. 1, no. 4, pp. 205-215.

https://doi.org/10.1016/j.invent.2014.08.003

Berger, T.; Hämmerli, K.; Gubser, N.; et al. (2011), 'internet-based treatment of depression: a randomized controlled trial comparing guided with unguided selfhelp,' Cognitive Behaviour Therapy, vol. 40, no. 4, pp. 251-266.

https://doi.org/10.1080/16506073.2011.616531

Berger, T.; Urech, A.; Krieger, T.; et al. (2017), 'Effects of a transdiagnostic unguided internet intervention ('velibra') for anxiety disorders in primary care: results of a randomized controlled trial,' Psychological Medicine, vol. 47, no. 1, pp. 67-80. https://doi.org/10.1017/S0033291716002270

Collins, S.; Byrne, M.; Hawe, J. \& O'Reilly, G. (2018), 'Evaluation of a computerized cognitive behavioural therapy programme, MindWise (2.0), for adults with mildto-moderate depression and anxiety,' British Journal of Clinical Psycholology, vol. 57, no. 2, pp. 255-269. https://doi.org/10.1111/bjc.12165

Communication COM/2018/233 final from the Commission to the European Parliament, the Council, the European Economic and Social Committee and the Committee of the Regions on enabling the digital transformation of health and care in the Digital Single Market; empowering citizens and building a healthier society, 25.4.2018.

de Graaf, L. E.; Gerhards, S. A.; Arntz, A.; et al. (2009), 'Clinical effectiveness of online computerised cognitive-behavioural therapy without support for depression in primary care: randomised trial,' The British Journal of Psychiatry, vol. 195, no. 1, pp. 73-80. https://doi.org/10.1192/bjp.bp.108.054429 
de Graaf, L. E.; Gerhards, S. A.; Arntz, A.; et al. (2011), 'One-year follow-up results of unsupported online computerized cognitive behavioural therapy for depression in primary care: A randomized trial,' Journal of Behavior Therapy and Experimental Psychiatry, vol. 42, no. 1, pp. 89-95.

https://doi.org/10.1016/j.jbtep.2010.07.003

Du, E.; Quayle, E. \& Macleod, H. (2013), 'Service providers' perceptions on the uptake of computerised cognitive behavioural therapy (CCBT),'PsychNology Journal, vol. 11, no. 3, pp. 213-233.

Gilbody, S.; Brabyn, S.; Lovell, K.; et al. (2017), 'On behalf of the REEACT collaborative telephone-supported computerised cognitive-behavioural therapy: REEACT-2 large-scale pragmatic randomised controlled trial,' The British Journal of Psychiatry, vol. 210, no. 5, pp. 362-367. https://doi.org/10.1192/bjp.bp.116.192435

Gilbody, S.; Littlewood, E.; Hewitt, C.; et al. (2015), 'On behalf of the REEACT Team. Computerised cognitive behaviour therapy (cCBT) as treatment for depression in primary care (REEACT trial): large scale pragmatic randomised controlled trial,' BMJ 11, 351, h5627. https://doi.org/10.1136/bmj.h5627

Hickie, I. B.; Davenport, T. A.; Luscombe, G. M.; et al. (2010), 'Practitionersupported delivery of internet-based cognitive behaviour therapy: evaluation of the feasibility of conducting a cluster-randomised trial,' Medical Journal of Australia, vol. 7, no. 192 (11 Suppl), pp. S31-S35.

Høifødt, R. S.; Lillevoll, K. R.; Griffiths, K. M.; et al. (2013), 'The clinical effectiveness of web-based cognitive behavioral therapy with face-to-face therapist support for depressed primary care patients: randomized controlled trial,' Journal of Medical internet Research, vol. 15, no. 8, e153. https://doi.org/10.2196/jmir.2714

Johansson, R.; Sjöberg, E.; Sjögren, M.; et al. (2012), 'Tailored vs. standardized internet-based cognitive behavior therapy for depression and comorbid symptoms: a randomized controlled trial,' PLoS One, vol. 7, no. 5, e36905.

Jonassaint, C.R.; Gibbs, P.; Belnap, B.H.; et al. (2017), 'Engagement and outcomes for a computerised cognitive-behavioural therapy intervention for anxiety and depression in African Americans,' BJPsych Open, vol. 3, no. 1, pp. 1-5. https:// doi.org/10.1192/bjpo.bp.116.003657

Kaltenthaler, E.; Brazier, J.; De Nigris, E.; et al. (2006), 'Computerized cognitive behavior therapy for depression and anxiety update: A systematic review and economic evaluation,' Health Technology Assessment, vol. 10, no. 33, pp. 1-70. https://doi.org/10.3310/hta10330

Kaltenthaler, E.; Parry, G. \& Beverley, C. (2004), 'Computerized cognitive behaviour therapy: A systematic review,' Behavioural and Cognitive Psychotherapy, vol. 32, no. 1, pp. 31-55. https://doi.org/10.1017/S135246580400102X 
Kaltenthaler, E.; Shackley, P.; Stevens, K.; et al. (2002), 'A systematic review and economic evaluation of computerised cognitive behaviour therapy for depression and anxiety,' Health Technology Assessment, vol. 6, no. 22, pp. 1-100. https://doi. org/10.3310/hta6220

Kay-Lambkin, F. J.; Baker, A. L.; Kelly, B. \& Lewin, T. J. (2011), 'Clinician-assisted computerised versus therapist-delivered treatment for depressive and addictive disorders: a randomised controlled trial,' The Medical Journal of Australia, vol. 195, no. 3, pp. S44-S50.

Kerikmäe, T.; Hamulak, O. \& Chochia, A. (2016), 'A historical study of contemporary human rights: deviation or extinction?’ Acta Baltica Historiae et Philosophiae Scientiarum, vol. 4, no. 2, pp. 98-115. https://doi.org/10.11590/abhps.2016.2.06

Kerikmäe, T. \& Joamets, K. (2018), 'Human dignity in Estonia,' in P. Becchi \& K. Mathis (eds.) Handbook of Human Dignity in Europe, Cham: Springer Verlag, pp. 1-16.

Kessler, D.; Lewis, G.; Kaur, S.; et al. (2009), 'Therapist-delivered internet psychotherapy for depression in primary care: a randomised controlled trial,' Lancet, vol. 374, no. 9690, pp. 628-634. https://doi.org/10.1016/S01406736(09)61257-5

Kivi, M.; Eriksson, M. C.; Hange, D.; et al. (2014), 'internet-based therapy for mild to moderate depression in Swedish primary care: short term results from the PRIMNET randomized controlled trial,' Cognitive Behaviour Therapy, vol. 43, no. 4, pp. 289-298. https://doi.org/10.1080/16506073.2014.921834

Knowles, S. E.; Toms, G.; Sanders, C.; et al. (2014), 'Qualitative meta-synthesis of user experience of computerised therapy for depression and anxiety,' PLoS One, vol. 9, no. 1, e84323. https://doi.org/10.1371/journal.pone.0084323

Layard, R. \& Clark, D. M. (2014), Thrive: The Power of Psychological Therapy, London: Penguin Books, Ltd.

Levin, W.; Campbell, D. R.; McGovern, K. B.; et al. (2011), 'A computer-assisted depression intervention in primary care,' Psychological Medicine, vol. 41, no. 7, pp. 1373-1383. https://doi.org/10.1017/S0033291710001935

Marks, I. M.; Cavanagh, K. \& Gega, L. (2007), 'Computer-aided psychotherapy: revolution or bubble?' The British Journal of Psychiatry, vol. 191, no. 6, pp. 471-473. https://doi.org/10.1192/bjp.bp.107.041152

Montero-Marín, J.; Prado-Abril, J.; Botella, C.; et al. (2015), 'Expectations among patients and health professionals regarding Web-based interventions for depression in primary care: a qualitative study,' Journal of Medical internet Research, vol. 17, no. 3, e67. https://doi.org/10.2196/jmir.3985

Newby, J. M.; Mackenzie, A.; Williams, A. D.; et al. (2013), 'internet cognitive behavioural therapy for mixed anxiety and depression: a randomized controlled trial and evidence of effectiveness in primary care,' Psychological Medicine, vol. 43, no. 12, pp. 2635-2648. https://doi.org/10.1017/S0033291713000111 
NICE (2011), Common Mental Health Problems: Identification and Pathways to Care, NICE Clinical Guidelines, no. 123, National Collaborating Centre for Mental Health (UK), Leicester: British Psychological Society.

Pennant, M. E.; Loucas, C. E.; Whittington, C.; et al. (2015), 'Computerised therapies for anxiety and depression in children and young people: A systematic review and meta-analysis,' Behaviour Research and Therapy, vol. 67, pp. 1-18. https://doi. org/10.1016/j.brat.2015.01.009

Perini, S.; Titov, N. \& Andrews, G. (2009), 'Clinician-assisted internet-based treatment is effective for depression: randomized controlled trial,' Australian \& New Zealand Journal of Psychiatry, vol. 43, no. 6, pp. 571-578. https://doi.org/10.1080/00048670902873722

Proudfoot, J.; Ryden, C.; Everitt; et al. (2004), 'Clinical efficacy of computerised cognitive behavioural therapy for anxiety and depression in primary care: randomised controlled trial,' The British Journal of Psychiatry, vol. 185, no. 1, pp. 46-54. https://doi.org/10.1192/bjp.185.1.46

Richards, D. \& Richardson, T. (2012), 'Computer-based psychological treatments for depression: a systematic review and meta-analysis,' Clinical Psychology Review, vol. 32, no. 4, pp. 329-342. https://doi.org/10.1016/j.cpr.2012.02.004

Rollman, B. L.; Herbeck, B. B.; Abebe, K. Z.; et al. (2017), 'Effectiveness of online collaborative care for treating mood and anxiety disorders in primary care: a randomized clinical trial,' JAMA Psychiatry, vol. 75, no. 1, pp. 56-64. https://doi.org/10.1001/jamapsychiatry.2017.3379

Ruwaard, J.; Schrieken, B.; Schrijver, M.; et al. (2009), 'Standardized web-based cognitive behavioural therapy of mild to moderate depression: a randomized controlled trial with a long-term follow-up,' Cognitive Behaviour Therapy, vol. 38, no. 4, pp. 206-221. https://doi.org/10.1080/16506070802408086

Salomonsson, S.; Santoft, F.; Lindsäter, E.; et al. (2017), 'Stepped care in primary care_-guided self-help and face-to-face cognitive behavioural therapy for common mental disorders: a randomized controlled trial,' Psychological Medicine, vol. 48, no. 10, pp. 1-11. https://doi.org/10.1017/S0033291717003129

Simblett, K. S.; Yates, M.; Wagner, A. P.; et al. (2017), Computerized cognitive behavioral therapy to treat emotional distress after stroke: A feasibility randomized controlled trial,' Journal of Medical internet Research JMIR Mental Health, vol. 4, no. 2, e16. https://doi.org/10.2196/mental.6022

Uusitalo, J. (2018), 'Protecting economic interests or the right to life? Perception of the European Court of Justice on emergency medical services,' Baltic Journal of European studies, vol. 8, no. 1, pp. 197-209. https://doi.org/10.1515/bjes-2018-0011

Waller, R. \& Gilbody, S. (2009), 'Barriers to the uptake of computerized cognitive behavioural therapy: a systematic review of the quantitative and qualitative 
evidence,' Psychological Medicine, vol. 39, no. 5, pp. 705-712.

https://doi.org/10.1017/S0033291708004224

WHO (2017), Depression and Other Common Mental Disorders: Global Health Estimates, Geneva: World Health Organization. Licence: CC BY-NC-SA 3.0 IGO. 